years for the total cohort, incidence of invasive PI in SLE was of 236/100.000 patient-years. As compared to the incidence in general French population, invasive $\mathrm{PI}$ was 26 times more frequent in SLE patients. PI occurred at a younger age $(43.5+14.9$ versus $65.3+18.7$ years, $p=0.009)$ and was more severe, with a higher frequency of invasive infection $(p<0.001)$ and higher need for ICU admission $(p=0.015)$ in SLE as compared to non SLE patients. Of note, unusual PI sites, including pneumococcal endocarditis $(n=1)$, arthritis $(n=1)$ and peritonitis $(n=1)$ were observed in SLE patients only. Risk factors associated with PI in SLE patients were a serum gammaglobulin level $<5 \mathrm{~g} / \mathrm{L}(\mathrm{p}=0.003)$ and a past history of lupus nephritis $(p=0.047)$, only. Steroids $(p<0.001)$ and immunosuppressive drugs $(p=0.027)$ were associated with infection severity.

Conclusions: Pneumococcal infections occur at a younger age, are more frequent and severe in SLE patients. Hypogammaglobulinemia and lupus nephritis increased the risk for $\mathrm{PI}$, whereas steroids and immunosuppressive drugs were associated with infection severity only. Our study shows that SLE patients have an increased risk for invasive $\mathrm{PI}$ and points to the need for vaccination against streptococcus pneumoniae in SLE.

Disclosure of Interest: None declared

DOI: 10.1136/annrheumdis-2017-eular.1016

\section{SAT0294 COMPARISON OF CLINICAL CARE BETWEEN CHINESE AND AMERICAN PATIENTS WITH SYSTEMIC LUPUS ERYTHEMATOSUS}

J. Dong ${ }^{1}$, H. Ma ${ }^{1}$, W.N. Roberts ${ }^{2}$, L. Wang ${ }^{1}$, F. Khan ${ }^{3}$, K.A. Lyn Shue ${ }^{2}$, L. Zhao ${ }^{1}$, L. Pan ${ }^{1} .{ }^{1}$ Rheumatology and Clinical Immunology, Affiliated Hospital of Qingdao University, Qingdao, China; ${ }^{2}$ Rheumatology; ${ }^{3}$ Internal Medicine, University of Louisville, Louisville, KY, United States

Background: In addition to gender and ethnicity, modifiable variables like geography, socioeconomic status, health system structure, education, and physician expertise may influence outcomes in systemic lupus erythematosus (SLE).

Objectives: To compare characteristics of and treatment options for subsets of Chinese and American patients with SLE to elucidate factors that contribute to disease activity and damage.

Methods: Chart review of 77 Chinese (Qingdao) and 48 Midwestern American (Louisville, Kentucky) patients meeting American College of Rheumatology (ACR) criteria for a diagnosis of SLE followed up for four years were analyzed retrospectively. Organ damage was assessed using the Systemic Lupus International Collaborating Clinics (SLICC)/ACR Damage Index (SDI), and disease activity was assessed using the Systemic Lupus Erythematosus Disease Activity Index (SLEDAI). Statistics were parametric exploratory tests of significance and multiple regression analyses in this hypothesis-generating effort.

Results: The interval between the time of onset and diagnosis was 44 months shorter in the Chinese arm $(p=0.001)$, and Chinese patients followed up at six times greater frequency than American patients $(p<0.001)$. Despite the lack of formal matching, the two cohorts featured similar disease activity according to the SLEDAI. Based on the SDI, rates of organ damage were higher in the American group. Chinese patients received more steroids, cyclophosphamide, hydroxychloroquine, intravenous immune globulin, and cyclosporine than the Louisville group, while the Louisville patients received more mycophenolate mofetil and azathioprine $(p=0.001)$.

\begin{tabular}{lccc} 
Table 1 & & \\
\hline Variable & $\begin{array}{c}\text { Qingdao }(n=77), \\
\text { mean } \pm \text { SD }\end{array}$ & $\begin{array}{c}\text { Louisville }(n=48), \\
\text { mean } \pm \text { SD }\end{array}$ & $p$-value \\
\hline Onset age (years) & $30.24 \pm 11.95$ & $30.21 \pm 12.21$ & 0.989 \\
Age at diagnosis (years) & $30.89 \pm 11.92$ & $34.5 \pm 12.99$ & 0.114 \\
$\begin{array}{l}\text { Duration between SLE onset and } \\
\quad \text { diagnosis (months) }\end{array}$ & $7.94 \pm 18.46$ & $52.30 \pm 89.90$ & 0.001 \\
Clinic visits per year & $10.93 \pm 7.09$ & $3.02 \pm 1.91$ & $<0.001$ \\
$\quad$ Interval between the last two times of & & & \\
$\quad$ follow up (months) & $1.89 \pm 1.31$ & $12.32 \pm 28.32$ & 0.014 \\
Disease duration (years) & $5.97 \pm 5.72$ & $5.22 \pm 5.53$ & 0.466 \\
SLEDAl & $5.81 \pm 4.32$ & $4.63 \pm 4.77$ & 0.156 \\
SDI & $0.44 \pm 0.64$ & $1.23 \pm 1.057$ & $<0.001$ \\
\hline
\end{tabular}

Table 2

\begin{tabular}{lccc}
\hline Medication & Qingdao $(n=77)$ & Louisville $(n=48)$ & $p$-value \\
\hline Prednisone & $77(100 \%)$ & $29(60.42 \%)$ & $<0.0001$ \\
Cyclophosphamide & $34(44.16 \%)$ & $6(12.50 \%)$ & $<0.0001$ \\
Hydroxychloroquine & $72(93.51 \%)$ & $32(66.67 \%)$ & $<0.0001$ \\
Methotrexate & $9(11.69 \%)$ & $3(6.25 \%)$ & 0.489 \\
Mycophenolate mofetil & $10(12.99 \%)$ & $18(37.50 \%)$ & 0.001 \\
Azathioprine & $2(2.60 \%)$ & $5(10.42 \%)$ & 0.147 \\
Intravenous immune globulin & $12(15.58 \%)$ & $1(2.08 \%)$ & 0.035 \\
Cyclosporine & $17(22.08 \%)$ & $1(2.08 \%)$ & 0.005 \\
\hline
\end{tabular}

Conclusions: The establishment of follow-up and treatment of SLE differs in specific, identifiable ways between these subsets of Chinese and midwestern American patients. Greater access to and increased frequency of follow-up appears associated with a lesser degree of organ damage, supporting the treat-to-target concept as applied to SLE. Complete, controlled trials in both settings are necessary, and further detailed comparison of larger cohorts may inform conclusions about the likelihood of generalizability of trial results from one setting to another.

Disclosure of Interest: None declared

DOI: 10.1136/annrheumdis-2017-eular.5561

\section{SAT0295 ANTI-RO52/TRIM21 ANTIBODIES ARE ASSOCIATED WITH QT INTERVAL PROLONGATION IN PATIENTS WITH SYSTEMIC LUPUS ERYTHEMATOSUS}

L.F. Perez, L.H. Silveira, L. Amezcua, O. Estevez, M. Moreno. Reumatologia, Instituto Nacional de Cardiologia Ignacio Chavez, Mexico City, Mexico

Background: Long QT syndrome (LQTS) is characterized by an abnormal QT corrected (QTC) interval prolongation that is associated with increased risk of sudden death. Studies have associated LQTS with several rheumatic conditions, and evidence points towards a link between the degree of systemic inflammation and the duration of QTc interval. Moreover, recent evidence suggests that anti-Ro antibodies may play a role in the QTc prolongation by mechanisms not fully understood, thus constituting a novel autoimmune-mediated LQTS.

Objectives: This study was aimed to assess whether QTc interval prolongation is associated with the presence of anti-Ro antibodies in SLE, particularly with reactivities against Ro52/TRIM21 antigens.

Methods: Consecutive patients fulfilling the 1997 ACR criteria for SLE were included. Patients with history of ischemic heart disease, with implantable pacemakers, and those taking drugs that potentially could affect QT interval (except for antimalarials) were excluded. Patients underwent a resting 12-lead electrocardiogram recording to measure QT interval corrected by BazzetÕs formula. A QTc interval duration greater than $460 \mathrm{msec}$ in women and $440 \mathrm{msec}$ in men was set to be abnormal. Serum anti-Ro and anti-Ro52/TRIM21 antibody levels were measured by ELISA. Data were expressed as frequencies and means ( \pm standard deviation), and differences were tested by YatesÕ continuity corrected chi square or Mann-Whitney tests, while linear regressions were performed to assess linearity between autoantibody levels and QTc duration. The GraphPad Prism 4.02 software was used for calculations.

Results: Sixty-six patients with mean age of $39 \pm 13$ years (57 female gender) were included. A QTc prolongation was found in 10 patients $(15 \%)$, with mean QTc interval of $470 \pm 18 \mathrm{msec}$ as compared to $414 \pm 23 \mathrm{msec}$ in those with no LQTS. Main clinical and demographic characteristics were similar for both groups, except for a lesser use of antimalarials and higher serum creatinine levels in patients with LQTS. Disease activity was similar between groups.Anti-Ro antibody levels were significantly higher in patients with prolonged QT interval ( $75 \pm 66 \mathrm{U} / \mathrm{mL}$ versus $29 \pm 44 \mathrm{U} / \mathrm{mL} ; \mathrm{P}=0.005)$; similarly, anti-Ro52/TRIM21 levels were higher in those with LQTS $(50 \pm 55 \mathrm{U} / \mathrm{mL}$ versus $14 \pm 30 \mathrm{U} / \mathrm{mL} ; P=0.01)$. Notably, a linear association (see the Figure) between the QTc intervals and levels of anti-Ro antibodies ( $\mathrm{r} 2=0.073 ; \mathrm{P}=0.02)$ and anti-Ro52/TRIM21antibodies $(\mathrm{r} 2=0.078 ; \mathrm{P}=0.02)$ was observed.
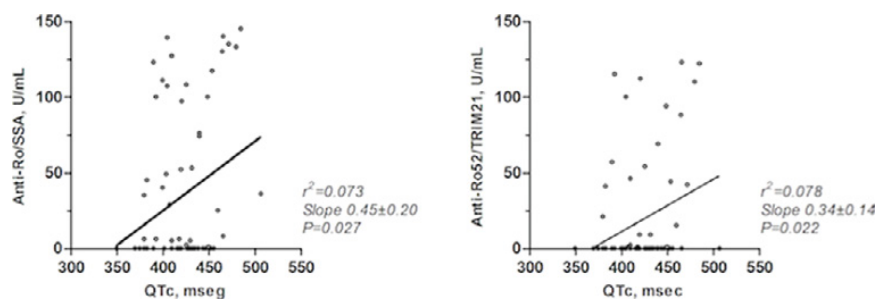

Conclusions: Our results strengthen the hypothesis that a specific autoantibodymediated LQTS occur in SLE patients positive to anti-Ro antibodies. This interference in the ventricular repolarization appears to be associated with increased levels of antibodies against Ro52/TRIM21 antigens, and supports the realization of an electrocardiogram as part of the routinely evaluation in SLE patient with circulating anti-Ro antibodies.

Disclosure of Interest: None declared

DOI: 10.1136/annrheumdis-2017-eular.6695

\section{SAT0296 RELATIONSHIP BETWEEN DISEASE ACTIVITY INDEX SCORES AND SUBJECTIVE ASSESSMENTS IN EARLY SYSTEMIC LUPUS ERYTHEMATOSUS}

M. Garabajiu ${ }^{1}$, V. Sadovici-Bobeica ${ }^{1}$, L. Mazur-Nicorici ${ }^{1}$, M. Cebanu ${ }^{1}$, V. Salaru ${ }^{2}$, M. Mazur ${ }^{1} .{ }^{1}$ Rheumatology; ${ }^{2}$ Family Medicine, State University of Medicine and Pharmacy "Nicolae Testemitanu", Chisinau, Moldova, Republic of

Objectives: To evaluate the disease activity in patients with early systemic lupus erythematosus (early SLE) and to compare it to patient's and physician's global assessment.

Methods: Cross-sectional study including 41 early SLE patients that fulfilled SLICC classification criteria, 2012. The early disease was defined one with the duration 2 years from the diagnosis. The disease activity was assessed by SLEDAI-2K and SLAM. Global indices were appreciated by patient and physician global assessments (PGA and MDGA), rated by $0-100$ numeric score. We correlated disease activity indices with global assessments by Pearson coefficient. 